\title{
SHAPE OPTIMIZATION OF UNDERGROUND EXCAVATION BY SIMULATED ANNEALING
}

\author{
MACIEJ SOBÓTKA, DARIUSZ ŁYDŻBA, ADRIAN RÓŻAŃSKI \\ Institute of Geotechnics and Hydrotechnics, Wrocław University of Technology, \\ Wybrzeże Wyspiańskiego 27, 50-370 Wrocław, Poland. \\ E-mail: maciej.sobotka@pwr.wroc.pl; dariusz.lydzba@pwr.wroc.pl, adrian.rozanski@pwr.wroc.pl
}

\begin{abstract}
The paper deals with the problem of shape optimization of underground tunnel excavation. In the pioneering work of Sałustowicz, the elliptic shape was assumed a priori to be an optimal one and then a determination of suitable semi-axes ratio was only the question. Two cases were distinguished there: self-supporting excavation and excavation with the structural support. An evolutionary structural optimization (ESO) procedure, employed in 2005 to optimize shape of underground excavation, confirms the assumptions concerning the case of self-supporting excavations. The case of supported excavation is considered in the present work. Optimization procedure of the simulated annealing (SA) is incorporated in the study. An energetic optimality condition, formulated in the authors' previous papers is also adopted. Itasca FLAC software is utilized in numerical examples. Four different in situ stress ratios are investigated. The numerical results obtained confirm that the optimal shape tends to be an ellipse. The semi-axes ratio demonstrates compliance with the assumptions already existing in literature.
\end{abstract}

\section{INTRODUCTION}

The problem of shape optimization of underground tunnel excavation appeared, according to the authors' knowledge, in literature in the 1960s in the work of Sałustowicz [7]. He assumed a priori the optimal shape to be an ellipse and derived the optimal ratio of semi-axes to be dependent on the ratio of horizontal to vertical insitu stress. Two different solutions were proposed, i.e., for supported and unsupported tunnel excavation, respectively. In the case of unsupported tunnel excavation, the following equation has been derived

$$
m_{\mathrm{opt}}=\left(\frac{a}{b}\right)_{\mathrm{opt}}=\frac{p_{x}}{p_{z}},
$$

where

$m_{\mathrm{opt}} \quad$ optimal ratio of the semi-axes of en ellipse,

$a, b \quad-$ lengths of semi-axes, horizontal and vertical, respectively,

$p_{x}$ and $p_{z}-$ in situ normal stresses, horizontal and vertical, respectively.

For the excavation with a structural support the optimal ratio of ellipse semi-axes is equal to the square root of in situ stress ratio 


$$
m_{\mathrm{opt}}=\left(\frac{a}{b}\right)_{\mathrm{opt}}=\sqrt{\frac{p_{x}}{p_{z}}} .
$$

In 2005, Ren et. al. [5] employed the evolutionary structural optimization (ESO) procedure [8], [9] to underground excavation optimization problem. This work confirms the results obtained by Sałustowicz involving the unsupported tunnel excavation. The shapes obtained were ellipse like and also the ratio of horizontal to vertical dimensions agrees with equation (1) given by Sałustowicz.

In 2009, the authors of present work formulated an energetic optimization condition for tunnel excavation with a support [2], [6]. Adopting the assumption proposed by Sałustowicz that the best shape is an ellipse, a series of numerical calculations have been performed. The results obtained demonstrate compliance with formula (2) given by Sałustowicz (2).

In the present paper, a heuristic, stochastic method called simulated annealing (SA) [3] with the energetic condition [6] as a cost function is employed to find optimal shape of supported excavation. No firm assumption concerning the shape is made. The obtained results presented here fully justify Sałustowicz's assumption that the optimal shape is an ellipse with semi-axes ratio governed by relation (2).

The paper is arranged as follows. After the introduction a formulation of the energetic condition is presented. The concept of simulated annealing is outlined in the next section. The numerical results of the four examples studied are presented in Section 5 . The examples examined differ with each other by the in situ stress ratio. A brief discussion concludes the paper.

\section{UNDERGROUND EXCAVATION SHAPE OPTIMIZATION CONDITION}

An energetic condition has been originally formulated for tunnel excavation with the structural support [6]. A crucial assumption, adopted in the formulation, is that the primary task of a support is to ensure the stability of excavation. In the case of tunnels at great depths, where the deformation pressure is the dominant load, this task is performed by retaining the convergence. Thus the following is assumed: the less convergence calculated with no support involved, the better the shape from the view of the support effort. In the original work [6], two measures of the convergence were postulated. The first one

$$
\Delta V=\int_{S} \overrightarrow{\mathbf{u}} \overrightarrow{\mathbf{n}} d S
$$

is a direct measure of change in the area of the tunnel cross-section due to the displacement of rock mass caused by the action of loads. The notation for quantities adopted in the above formula is presented in Fig. 1. 


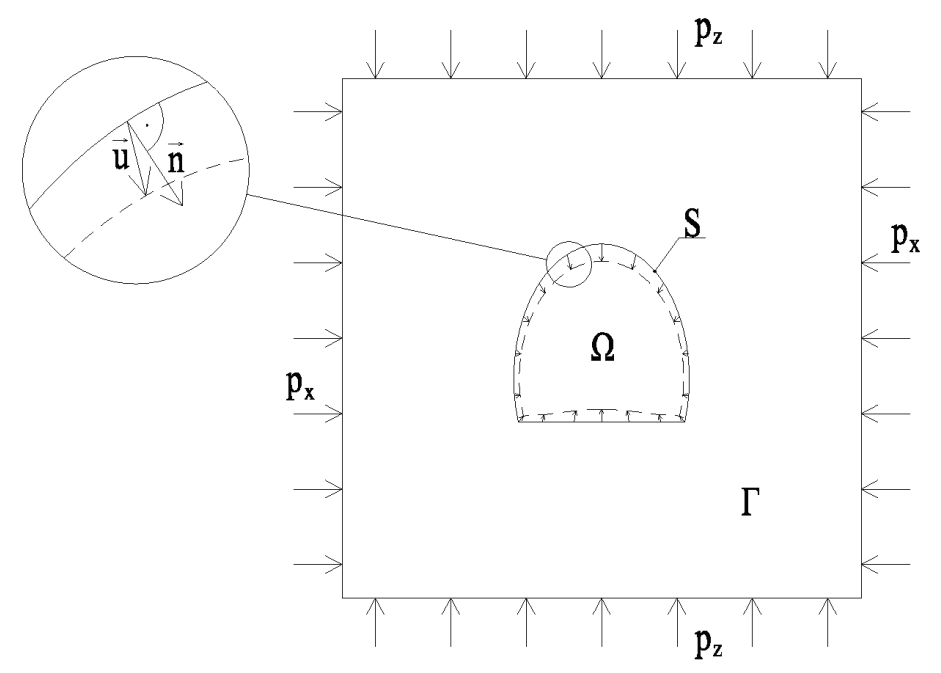

Fig. 1. Adopted designation: $\Gamma, \Omega$-rock mass and excavation area, respectively; $S$ - excavation contour, $\overrightarrow{\mathbf{u}}$ - displacement vector, $\overrightarrow{\mathbf{n}}$ - unit normal vector

The other proposed measure of convergence is the elastic volumetric strain energy cumulated in the material of tunnel core (region $\Omega$ ) if it would be subjected to displacements $\overrightarrow{\mathbf{u}}$, taken as a boundary condition. Then the measure is defined as

$$
E_{0}=\frac{1}{2} K \int_{\Omega}\left(\varepsilon_{x}+\varepsilon_{z}\right)^{2} d \Omega
$$

where

$K$ - denotes the bulk modulus of the rock mass,

$\varepsilon_{x}, \varepsilon_{y}-$ are the values of normal strain components horizontal and vertical, respectively.

The second measure (4) has a few advantages: it is positively defined and easier to implement in numerical calculations. Thus the measure (4) is finally postulated as the optimization condition.

In paper [6], a series of numerical calculations performed to test the optimality condition are presented. Three series of calculations for different values of horizontal to vertical pressure ratios, $p_{x} / p_{z}=1, p_{x} / p_{z}=1 / 4$ and $p_{x} / p_{z}=1 / 9$ have been carried out. Finite element (FE) analysis using FlexPDE has been performed. Material constants for rock mass region $\Gamma$ have been assumed as: Young's modulus $E=7 \mathrm{e} 10 \mathrm{~Pa}$ and Poisson's ratio $v=0.26$. An assumption of Sałustowicz has also been adopted the optimal shape is elliptic. The constant cross-section area of a tunnel excavation has been required in addition. With such limitation the only variable parameter of 
a shape was the semi-axes ratio $m$. For each particular $p_{x} / p_{z}$ value a sequence of numerical calculations for different $m$ ratio values were carried out. The optimal semi-axes ratio $m_{\text {opt }}$ has been numerically proved as the one that minimizes the value of $E_{0}$.

The obtained results, in all three calculation series, show that the consequence of taking $E_{0}$ as an objective function is equivalent to using equation (2). In this paper, a novel method of tunnel excavation optimization, that does not require any strong assumption concerning the excavation shape, is incorporated. The basis of the method proposed is the simulated annealing procedure. A concept of the method is outlined in the next section.

\section{SIMULATED ANNEALING}

Simulated annealing [3] is a heuristic, stochastic method of optimization. The basis of the procedure derives from physical process in metallurgy called annealing. It involves the heating of material to above its critical temperature, maintaining at suitable temperature during some time interval and then cooling with prescribed slow enough rate. At high temperatures the rearrangement of particles is a continuous, chaotic process, described in terms of statistical mechanics. During the cooling the movements of the particles become less chaotic, the microstructure is gradually refined. If the cooling is slow enough the particles eventually set at low energy levels and the system reaches a stable state, optimal in the sense of the value of the internal energy.

A simple algorithm for simulation of a collection of atoms at a given temperature is proposed in [4]. A system is brought to equilibrium by an iterative procedure. In each iteration an atom is given a random displacement. If the energy of the system in the new configuration is less than in the previous one $(\Delta E<0)$, the change is accepted, otherwise the change is accepted with the probability

$$
P(\Delta E)=e^{-\frac{\Delta E}{k_{B} T}},
$$

where $k_{B}$ is the Boltzmann constant and $T$ is the actual temperature.

An analogous procedure is adopted in a single iteration of simulated annealing. The internal energy is replaced by a suitable cost function and the temperature is an artificial parameter controlling the randomness of the process. When the temperature decreases the system becomes less chaotic. Finally, the system is brought to the stable state. Simultaneously the cost function reaches a minimum. The procedure of shape optimization of excavation by simulated annealing is dealt with in detail in the next section. 


\section{A PROCEDURE OF EXCAVATION SHAPE OPTIMIZATION}

The system under consideration is a plain boundary value problem being a model of an underground tunnel excavation in the rock mass at some depth. A commercially available code FLAC 7.00, based on finite difference method, is utilized to solve a series of boundary value problems. Only a quarter of an area around the excavation is modelled, due to the symmetry. The region of the rock mass is a square of side length $100 \mathrm{~m}$. The starting point of en excavation shape (to be then optimized) is a square of side length $10 \mathrm{~m}$. A static scheme of the problem is presented in Fig. 2 .

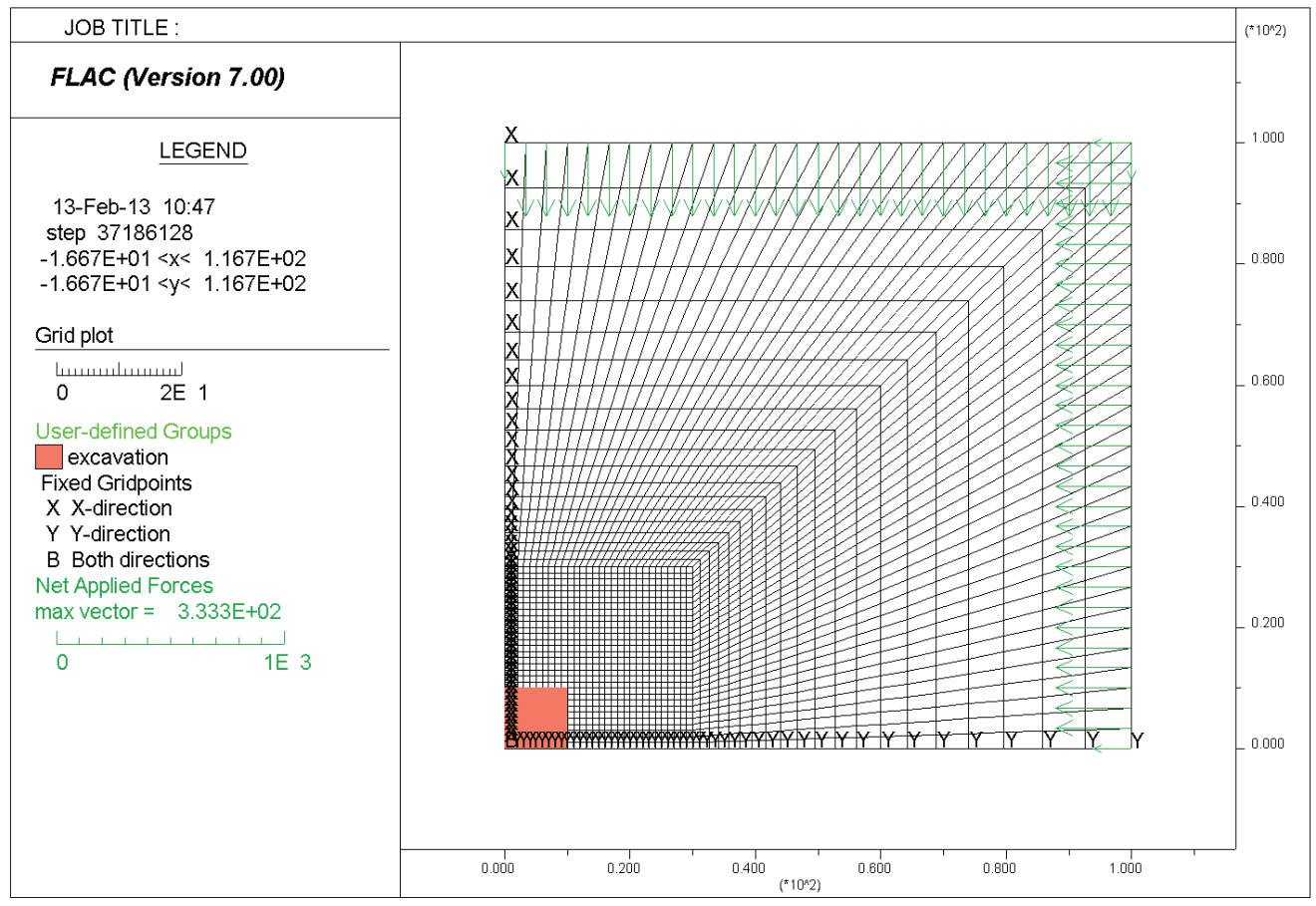

Fig. 2. A boundary value problem under consideration

Boundary conditions are as follows: the displacements perpendicular to the truncations at the symmetry lines are set to be null whereas at the other truncation lines the uniform values of normal stresses equal to in situ stress are assumed. A linear elastic model of the material is adopted. The parameters of the rock mass are: bulk modulus $K=1 \mathrm{e} 8 \mathrm{~Pa}$ and shear modulus $G=1 \mathrm{e} 8 \mathrm{~Pa}$. The parameters adopted for the region of excavation are $K=1 \mathrm{e} 2 \mathrm{~Pa}$ and $G=1 \mathrm{e} 2 \mathrm{~Pa}$, so that the stiffness of an excavation region is negligible compared to the stiffness of the rock mass. 
The cost function adopted in the problem of shape optimization is the energy $E_{0}$ defined by equation (4). A single permissible change in the shape of excavation in one single iteration is a swap of two zones of different material, so that the area of an interior of excavation must remain simply connected space. In other words, the excavation region must never divide into two or more separate regions and must not have the "islands" of the rock mass region. Use of such procedure for a single change in the shape does not allow for any change of the area of the excavation region. Notice that the swaps are admissible only in a range of the regular grid of $30 \times 30$ zones (see Fig. 2).

At a given temperature level a hundred of iteration steps are executed. A simple iteration consists of:

- a random choice of a simple change in shape of the excavation,

- calculation of a change in the energy $\Delta E_{0}$,

- if a change causes a decrease of energy $E_{0}$, i.e., $\Delta E_{0}<0$ (the shape becomes better) the change is accepted, else a uniformly distributed random number $p$ over an interval between 0 and 1 is generated. Then, if

$$
p<P\left(\Delta E_{0}\right)=e^{-\frac{\Delta E_{0}}{T}},
$$

the change is accepted otherwise it is not.

A temperature $T_{0}$ at the start of the optimization procedure is determined in such a way that implies the probability of acceptance of a change in the case of $\Delta E>0$ being equal to 0.5 . To estimate the starting temperature $T_{0}$ the average $\overline{\Delta E_{0}}$ for one thousand random possible changes in starting shape is determined. Then the temperature $T_{0}$ is derived from inequality (6) by substitutions $p=0.5, \Delta E_{0}=\overline{\Delta E_{0}}, T=T_{0}$. Finally, the starting temperature is

$$
T_{0}=-\frac{\overline{\Delta E_{0}}}{\ln 0.5} .
$$

After every one hundred iterations the temperature is decreased. The cooling procedure is performed as a geometric sequence, i.e., a new temperature is 0.9 times of the previous one. The optimization procedure ends when further decreasing of the temperature does not make any difference in the excavation shape.

\section{NUMERICAL EXAMPLES}

The procedure is executed for four different ratios of in situ stress, namely $p_{x} / p_{z}=$ $1, p_{x} / p_{z}=1 / 2, p_{x} / p_{z}=1 / 3, p_{x} / p_{z}=1 / 4$. The shapes of excavation obtained after 1000 , 
2000, 3000, 4000, 5000 and 6000 iterations, in all four cases studied, are presented in Figs. 3-6, respectively. Note that not the full range of boundary value problem is shown but only the regular section of the grid where the swaps of zones (changes in excavation shape) are permissible.
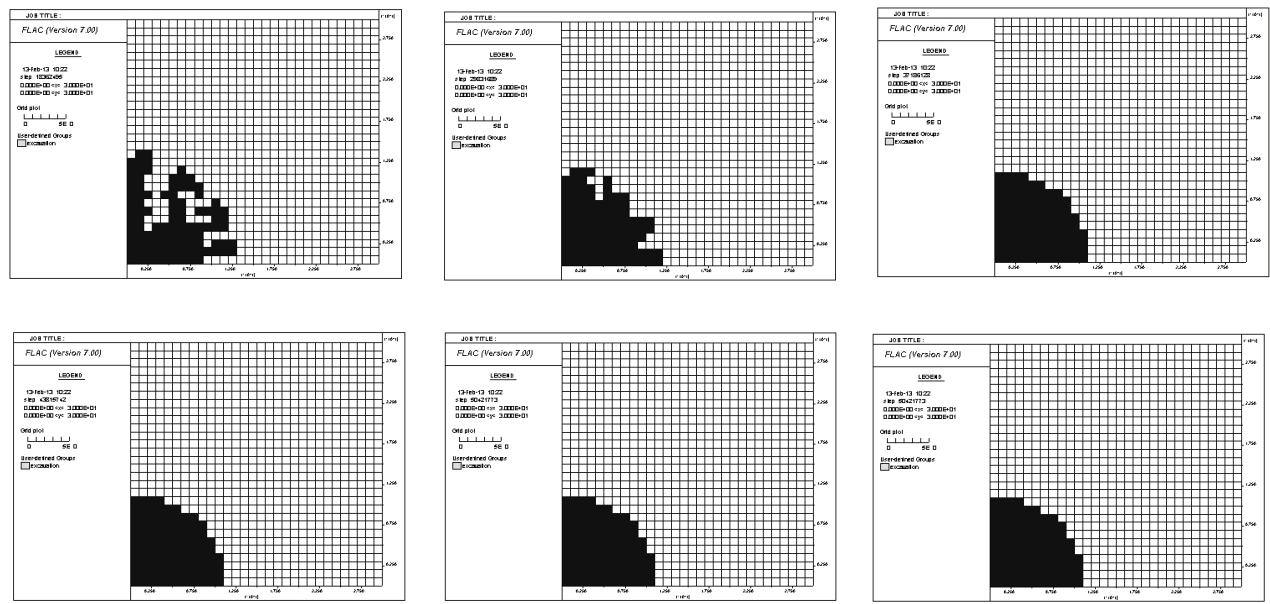

Fig. 3. Shapes of excavation after 1000, 2000, 3000, 4000, 5000 and 6000 iterations for $p_{x} / p_{z}=1$
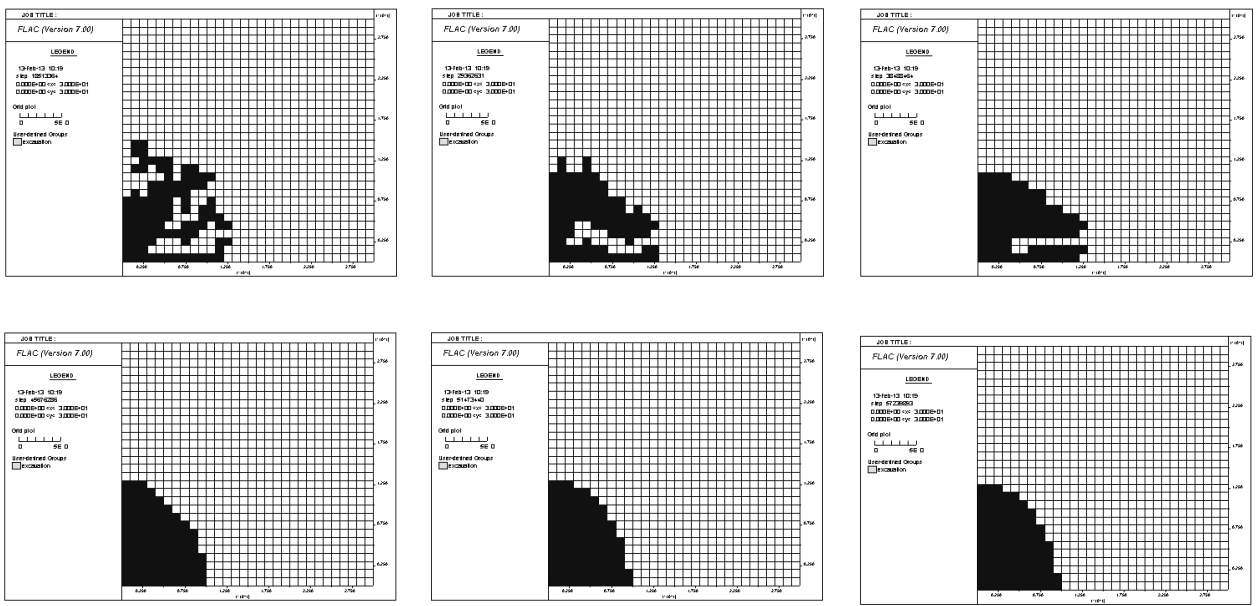

Fig. 4. Shapes of excavation after 1000, 2000, 3000, 4000, 5000 and 6000 iterations for $p_{x} / p_{z}=1 / 2$ 

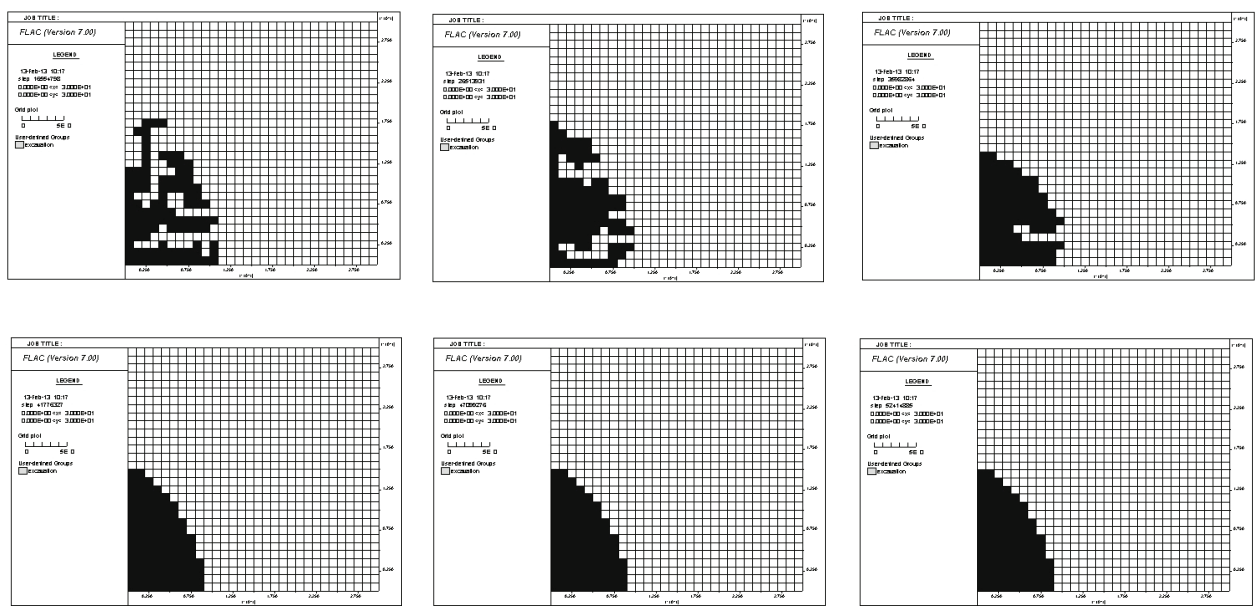

Fig. 5. Shapes of excavation after 1000, 2000, 3000, 4000, 5000 and 6000 iterations for $p_{x} / p_{z}=1 / 3$
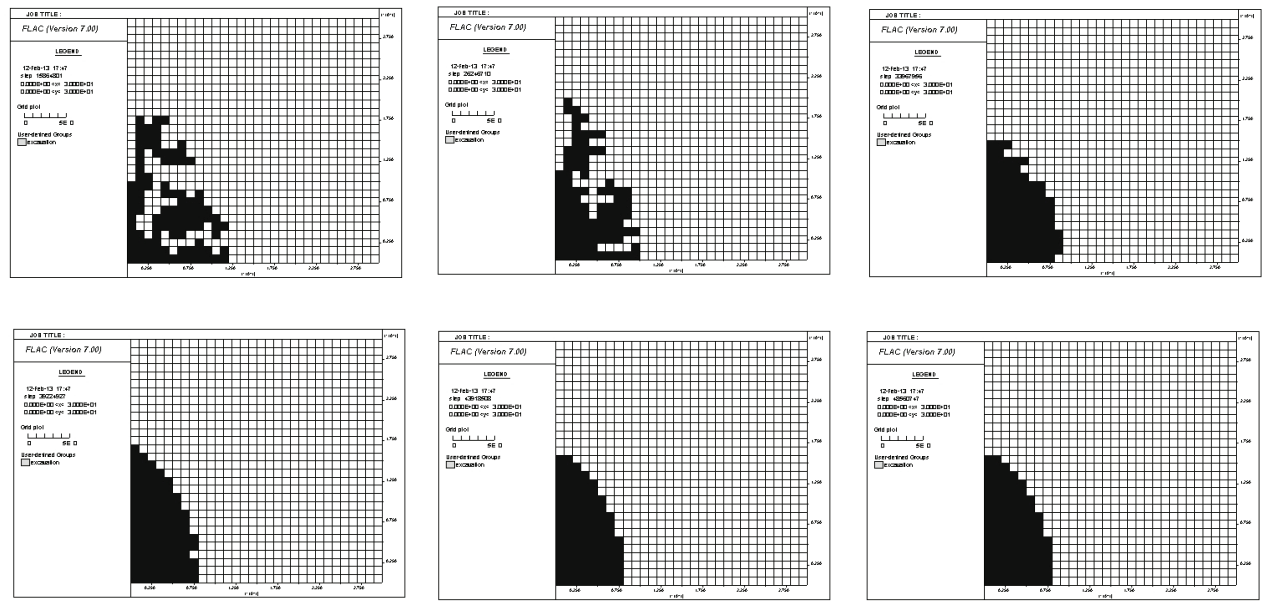

Fig. 6. Shapes of excavation after 1000, 2000, 3000, 4000, 5000 and 6000 iterations for $p_{x} / p_{z}=1 / 4$

\section{DISCUSSION OF RESULTS}

In all the cases of in situ stress the shape stabilizes after approximately 5000 iterations, which corresponds to final temperature $T=0.9^{50} T_{0}$. The results obtained justify the Sałustowicz assumption: the optimal shape, in the view of support effort, tends to an ellipse with the ratio of semi-axes given by equation (2). Figure 7 illustrates 
that fact in detail. It presents the shapes obtained by simulated annealing in comparison with the ellipses of semi-axes ratio postulated by Sałustowicz. All four cases considered, i.e., $p_{x} / p_{z}=1, p_{x} / p_{z}=1 / 2, p_{x} / p_{z}=1 / 3, p_{x} / p_{z}=1 / 4$ are presented, from the left to the right, respectively.
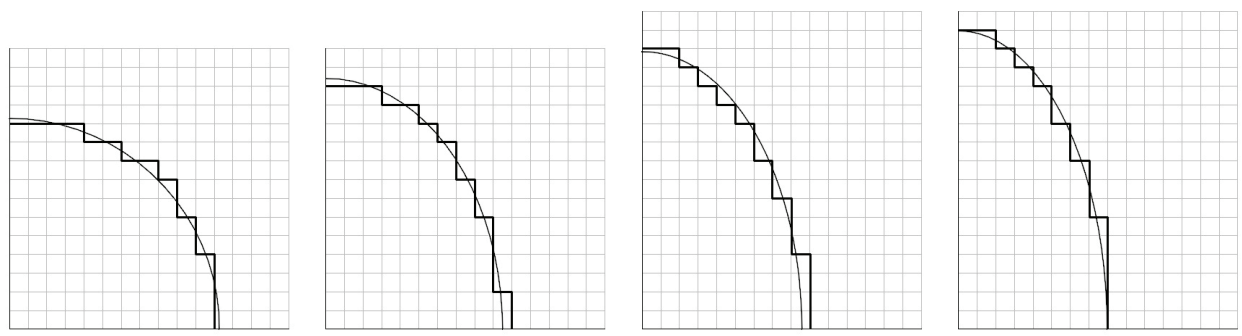

Fig. 7. The shapes obtained in comparison with the elliptic shapes postulated by Sałustowicz; $p_{x} / p_{z}=1, p_{x} / p_{z}=1 / 2, p_{x} / p_{z}=1 / 3, p_{x} / p_{z}=1 / 4$ from the left to right

\section{CONCLUSIONS}

A shape optimization by simulated annealing is presented. The starting shape of a square of $10 \times 10$ zones, reflecting the quarter of excavation, is gradually converted to the optimal one in accordance with the procedure routines. Four different in situ stress states are considered in numerical examples. No firm assumption concerning the shape of tunnel excavation is postulated a priori. The shape consists of a fixed number of finite difference zones (pixels) and must remain simply connected space during the optimization process. The results justify the assumption proposed by Sałustowicz, i.e., the optimal shape tends to be an ellipse. Also the condition for the semi-axes length ratio is consistent with the one proposed by Sałustowicz.

\section{REFERENCES}

[1] FLAC Fast Lagrangian Analysis of Continua, User's Guide, Itasca Consulting Group Inc. Minneapolis, 2011.

[2] KaWA M., RÓŻAŃSKi A., SoBÓtKA M., A verification of shape optimization procedures of tunnel underground excavations, (in Polish), Górnictwo i Geoinżynieria, Book 2, 2011, 535-541.

[3] Kirkpatrick S., Gelatt C., Vecchi M., Optimization by simulated annealing, Science, 1983, Vol. 220, No. 4598, 1983, 671-680.

[4] Metropolis N. et al., Equation of state calculation by fast computing machines, The Journal of Chemical Physics, Vol. 21, No. 6, 1953, 1087-1092.

[5] Ren G., Smith J.V., TANG J.W., XIE Y.M., Underground excavation shape optimization using an evolutionary procedure, Computers and Geotechnics, No. 32, 2005, 122-132. 
[6] RÓŻAŃSKi A., SoBótKA M., A procedure of underground excavations shape optimization, (in Polish), Górnictwo i Geoinżynieria, Book 1, 2009, 519-529.

[7] SaŁustowicz A., Zarys mechaniki górotworu, Wydawnictwo Śląsk, Katowice, 1968.

[8] XIE Y.M., Steven G.P., A simple evolutionary procedure for structural optimization, Comput. Struct., No. 49(5), 1993, 885-896.

[9] XIE Y.M., Steven G.P., Evolutionary structural optimization, Springer, Berlin, 1997. 